\title{
Mathematical modeling of the formation of sedimentary acid precipitation in the atmosphere in view of the evaporation of moisture from their surface
}

\author{
Dmitry Gvozdyakov ${ }^{1, *}$, Vladimir Gubin ${ }^{1}$, Svetlana Shvab ${ }^{1}$, Anastasia Sidorova ${ }^{1}$, and \\ Nikolay Semenov ${ }^{1}$ \\ ${ }^{1}$ National Research Tomsk Polytechnic University, 634050 Tomsk, Russia
}

\begin{abstract}
The article presents the results of numeric simulation of the formation of sedimentary acid precipitation in the atmosphere taking into account the evaporation of moisture from their surfaces. It is established that the joint condensation of vapors of sulfuric anhydride and water vapor, given the flow of solar energy and the evaporation process significantly slows the growth of drops. The possibility of achieving the underlying surface by the formed sediments is analyzed.
\end{abstract}

\section{Introduction}

The Earth's atmosphere is a composite of many gaseous compounds of natural [1] (oxygen, nitrogen, etc.) and anthropogenic (oxides of nitrogen, sulfuric anhydride, etc.) origin [1-4].

It is known [5] that the geometric characteristics of atmospheric droplets repeatedly changed in the process of movement. When the droplet diameter $(d)$ from $10^{-6} \mathrm{~m}$ to $100 \cdot 10^{-}$ ${ }^{6} \mathrm{~m}$, typically, the droplets have a spherical shape [6], if $100 \cdot 10^{-6}<d<1 \cdot 10^{-3} \mathrm{~m}$ of the ellipsoid. Based on the results of [6] we can assume that the geometric parameters of the droplets will affect the area of their deposition on the Earth's surface, as the motion of droplets of non-spherical shape different from the spherical movement not only quantitative characteristics (higher values of coefficient of drag $-C_{D}$ ), but the mechanism of movement.

Previous investigations [7-8] illustrate the mechanism of formation of sedimentary acid precipitation in the atmosphere in areas of thermal power stations location without considering the process of evaporation of moisture from their surface.

The aim of this work is mathematical modeling of the formation of sedimentary acid precipitation of spherical shape in the atmosphere in view of the "moderate" evaporation and the major significant factors.

We used the iterative algorithm developed for solving nonlinear heat conduction problems in a local heat exposure [9] method of finite differences.

\footnotetext{
* Corresponding author: dim2003@.tpu.ru
} 
According to [6], the influence of non-sphericity coefficient drops characterized nonsphericity G, as determined ratio of the surface area of a real drop in the area of similar size sphere.

\section{Mathematical problem}

Heat equations drops and movement within the accepted physical model of the test process is as follows:

$$
\left.\begin{array}{c}
\rho_{1} \cdot C_{P} \cdot \frac{\partial T_{1}}{\partial t}=\lambda\left(\frac{\partial^{2} T_{1}}{\partial r^{2}}+\frac{2}{r} \cdot \frac{\partial T_{1}}{\partial r}\right) \\
\frac{d U_{X}}{d t}=\frac{3 \cdot G \cdot C_{D} \cdot \rho_{1} \cdot\left(U_{X}-U_{0}\right) \cdot\left(U_{X}-U_{0}\right)}{8 \cdot \pi \cdot \delta \cdot \rho_{2}} \\
\frac{d V_{Y}}{d t}=g+\frac{3 \cdot G \cdot C_{D} \cdot \rho_{1} \cdot\left(V_{Y}-V_{0}\right) \cdot\left(V_{Y}-V_{0}\right)}{8 \cdot \pi \cdot \delta \cdot \rho_{2}}
\end{array}\right\}
$$

where $\rho_{1,2}$ - density, $\mathrm{kg} / \mathrm{m}^{3} ; C_{P}$ - isobaric heat capacity, $\mathrm{J} /(\mathrm{kg} \cdot \mathrm{K}) ; \lambda$ - coefficient of thermal conductivity, $W /(m \cdot K) ; T$ - temperature, $K ; t$ - time, $s ; V_{X}, V_{Y}$ - drop velocity in the direction of the axes $x$ and $y$, respectively, $\mathrm{m} / \mathrm{s} ; C_{D}$ - drag coefficient; $g$ - acceleration of gravity, $\mathrm{m} / \mathrm{s}^{2} ; U_{0}, V_{0}$ - velocity components ambient, $\mathrm{m} / \mathrm{s}$.

The initial and boundary conditions are:

$$
t=0: T=T_{0}, 0 \leq r \leq \delta,
$$

$$
r=\frac{\delta}{2}: \quad-\lambda_{1} \cdot \frac{\partial T_{1}}{\partial r}+Q \cdot\left(W_{K}^{S O_{3}}+W_{K}^{\text {steam }}\right)-P=-\lambda_{2} \cdot \frac{\partial T_{2}}{\partial r}, \quad T_{1}=T_{2} ; \quad r=0: \quad \frac{\partial T_{1}}{\partial r}=0,
$$

where $T_{1}, T_{2}$ - the temperature drops and a gaseous medium, respectively, $K$; $W_{K}^{S O_{3}}, W_{K}^{\text {steam }}$ - mass condensation rate, $\mathrm{kg} /\left(\mathrm{m}^{2} \cdot \mathrm{s}\right) ; Q$ - the heat of reaction, $\mathrm{J} / \mathrm{kg} ; \delta$ and $\mathrm{r}-$ the characteristic dimensions of the drops, $m ; P$ - density of solar energy flux, $\mathrm{W} / \mathrm{m}^{2}$.

Mathematical modeling performed at the following values of the parameters characterizing the initial conditions of the considered process: $U_{0}=5 \mathrm{~m} / \mathrm{s} ; V_{0}=0.5 \mathrm{~m} / \mathrm{s}$; temperature drops at the initial moment of time: summer $-294 \mathrm{~K}$; winter $-262 \mathrm{~K}$. Investigations of the influence of geometrical characteristics of emerging acid drops are made for range of time from $6000 \mathrm{~s}$ to the surface of the condensation nuclei sized from $5.00 \cdot 10^{-6} \mathrm{~m}$.

In Fig. 1, 2 shows the trajectory of the droplets in the air space in winter and summer.

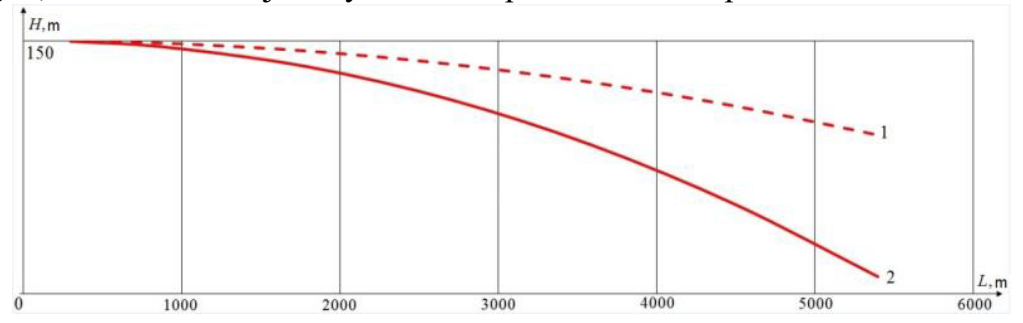

Fig. 1. The trajectory of the drops during the summer: 1 - taking into account the process of evaporation; 2 - without taking into account the process of evaporation. 


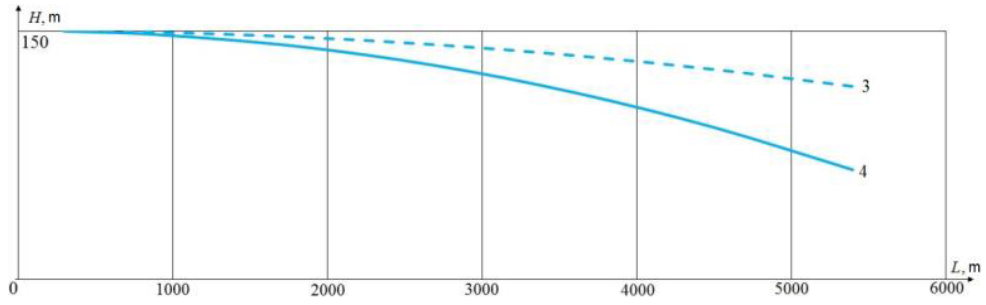

Fig. 2. The trajectory of the drops in winter: 3 - taking into account the process of evaporation; 4 without taking into account the process of evaporation.

Two thermal periods are analyzed, typical for regions with sharply continental climate given the mechanism of moisture evaporation from the surface of the forming precipitation. The value of the flux density of solar energy was taken equal to the value of the solar constant.

The simulation results show that the trajectory of the particles change significantly given the mechanism of moisture evaporation from the surface of the forming precipitation. It is established that in summer, during the time interval, precipitation are formed that are unable to reach the underlying surface in a given radius $(6000 \mathrm{~m}$ from the emission source, located at the height of $150 \mathrm{~m}$ ). Atmospheric drops in this period of the year under given settings and conditions are to be at the height of 120-150 m from the underlying surface. A similar pattern is observed in the winter (see Fig. 2, feature 3).

\section{Conclusion}

Analysis of the results of mathematical modeling of process of formation of sedimentary acid precipitation leads to the conclusion that co-condensation of vapor of sulfuric anhydride and steam with account for the flow of solar energy and evaporation of moisture from the surface drops significantly (up 40\%) retards the growth drops. The resulting precipitation in the studied radius with given parameters are not reached the underlying surface.

The research has been accomplished with financial support of the Russian Federation Ministry of Education and Science within implementation of the project №13.7644.2017/BCH.

\section{References}

1. G.E. Zaikov, S.A. Maslov, V.L. Robalo, Acid rain and the environment (Chemistry, Moscow, 1991)

2. E.P. Volkov, E.I. Gavrilov, F.P. Dugih, Flue pipe TPP and NPP (Energoatomizdat, Moscow, 1987)

3. K.V. Slyusarskiy, K.B. Larionov, V.I. Osipov, S.A. Yankovsky, V.E. Gubin, A.A. Gromov, Fuel, 191, 383 (2017)

4. K.V. Slyusarskiy, A.V. Zenkov, S.A. Yankovsky, A.A. Matveeva, S.V. Lavrinenko, A.A. Gromov, MATEC Web Conf. 72 (2016)

5. Y.E. Geguzin, Drop (Science, Moscow, 1973)

6. V.I. Terekhov, M.A. Pakhomov, Heat and mass transfer and hydrodynamics in gasdrop flows, (Novosibirsk, NSTU, 2008)

7. D.V. Gvozdyakov, V.E. Gubin, Chem. Pet. Eng. 51 (3), 201 (2015)

8. D.V. Gvozdyakov, V.E. Gubin, S.A. Shvab, A.O. Tanishev, EPG Web Conf. 72 (2016)

9. G.V. Kuznetsov, M.A. Sheremet, Russ. Microelectron. 37 (2), 131 (2008) 